\title{
Cost benefit analysis of distribution network using micro generation
}

\author{
A. Srinivasa Rao ${ }^{1 *}$, R. Gowrisankara Rao², S.V. L. Narasimham ${ }^{3}$ and R. Srinivasa Rao ${ }^{4}$ \\ ${ }^{1}$ Department of Electrical and Electronics Engineering, GITAM University, Visakhapatnam-530045, India \\ ${ }^{2}$ Department of Electrical and Electronics Engineering, MVGR College of Engineering, Vizayanagaram-535005, India \\ ${ }^{3}$ Department of Information Technology, JNT University, Hyderabad-500085, India \\ ${ }^{4}$ Department of Electrical and Electronics Engineering, JNT University, Kakinada-533003, India \\ asrao47@yahoo.com
}

\begin{abstract}
Energy consumption in India is rising rapidly mainly due to growth in economy and population. The only alternative to meet the ever rising demands of the nation is to tap the potential of renewable energy sources and promote Micro generation at the consumer's end. This approach ensures a greener energy with an added benefit of minimized transmission losses. In this paper an attempt has been made to envisage the impact of micro generation at the consumer's premises using renewable sources like solar and wind. In this approach only solar power is considered for computations which are injected during peak and off peak hours in order to reduce the power loss, thus improving the quality of power. This results in reduction of fossil fuel consumption. Power losses of various distribution networks are computed and the results are presented and analyzed. Cost benefit analysis is also carried out on two networks namely IEEE and Andhra Pradesh Eastern Power Distribution Corporation Limited (APEPDCL) to obtain the payback period of the investment.
\end{abstract}

Keywords: Power loss, Micro Generation, Cost Benefit Ratio and Distributed Generation

Nomenclature: LP (i) and LQ (i): real and reactive power at bus i; R (i) and X (i): Resistance and Reactance connected to bus i; $P(i+1)$ and Q (i+1): Real and reactive power at bus $i+1 ; \mathrm{V}(\mathrm{i}+1)$ : Voltage at bus $i+1$; LB (i,k): Node just ahead of source node of $\mathrm{i}^{\text {th }}$ node and $\mathrm{k}^{\text {th }}$ lateral; $\mathrm{P}_{\mathrm{G} i+1}$ : Generated power; DY: Sunny days available per year; H: Sunny hours available per day.

\section{Introduction}

Indian power sector is passing through its most difficult phase as the demand for power is accelerating at a rate which the supply is unable to cope up, resulting in high levels of energy shortage both at peak and base levels. The country's annual electricity generation capacity has increased in last 20 years by about $120 \mathrm{GW}$, from about $66 \mathrm{GW}$ in 1991 to over $100 \mathrm{GW}$ in 2001 , to over 185 GW in 2011 [CEAMP,2011]. Base load requirement was 861,591 (MU) against availability of 788,355 MU which results in 8.5\% deficit. During peak loads, the demand was $122 \mathrm{GW}$ against the available $110 \mathrm{GW}$ which stands at $9.8 \%$ deficit (Ministry of power, 2011).

If current average transmission and distribution losses remain same (23\%), India needs to add about $135 \mathrm{GW}$ of power generation capacity, before 2017, to satisfy the projected demand after losses. The ministry of Non conventional Energy Sources (MNES) is taking steps to meet a part of the above demand by tapping different sources of renewable energy like solar, wind, small hydro, biomass etc. Out of these sources, the solar energy is preferred as it is pollution free, economical and has lesser maintenance cost. Moreover, solar energy is available for at least 8 hours a day for over 300 days in most parts of India and hence it is considered. It can be connected to the grid to reduce grid overloading at peak demand [Sethi and Nitin, 2009]. In this paper solar power is considered for Micro Generation to meet the demand of energy and it is performed on two networks viz. IEEE standard network and practical EPDCL networks. The Distributed Generation approach is taken into account to reduce the losses

\section{Proposed method}

Distribution load flow has been implemented to compute power loss and voltage profile of a given distribution network (Chen et al.,1981; Electrical World,1986; Vempati et al.,1987). In the proposed method an attempt has been made to compare different systems with and without Micro Generation.

Power loss is computed for each bus of the given network as follows:

$\mathrm{LP}(\mathrm{i})=\mathrm{R}(\mathrm{i}) *\left[\mathrm{P}(\mathrm{i}+1)^{2}+\mathrm{Q}(\mathrm{i}+1)^{2}\right] / \mathrm{V}(\mathrm{i}+1)^{2}$

LQ (i) $=\mathrm{X}(\mathrm{i}) *\left[\mathrm{P}(\mathrm{i}+1)^{2}+\mathrm{Q}(\mathrm{i}+1)^{2}\right] / \mathrm{V}(\mathrm{i}+1)^{2}$ 
For the same network solar power is generated and injected at every bus and compute the voltage at each bus and power loss of given network. To calculate average injected power to a network the following formula is adopted:

$\begin{array}{ll}\mathrm{P}_{\mathrm{av} i+1}=\mathrm{P}_{\mathrm{Gi}+1} * \mathrm{DY} * \mathrm{H} / \mathrm{T} & \text { (i) } \\ \mathrm{P}_{\mathrm{B} \mathrm{i}+1}=\mathrm{P}_{\mathrm{Li}+1}-\mathrm{P}_{\mathrm{av} i+1} & \text { (ii) }\end{array}$

The power generated from Solar panels is converted into average injected power using equation (i) and get the new values of bus power based on equation (ii). Compute voltage profile and power loss with new bus powers using load flow algorithm. Now calculate reduction in power loss and voltage profile by comparing base case with Micro Generation

\subsection{Algorithm for proposed approach:}

\section{Step 1: Start}

Step 2: Read line and load data of radial distribution network

Step 3: Initialize branch power losses LP (i) and LQ (i) to zero from $i=1$ to EN-1

Step 4: Set iteration count IT=1

Step 5: Initialize total active and reactive power losses to zero and assume source node voltage as $1 \mathrm{p}$.u. and set convergence value as 0.0001 .

Step 6: Set $\mathrm{i}=0$ and compute $\mathrm{P}(\mathrm{i}+1), \mathrm{Q}(\mathrm{i}+1)$ from equations

$$
\begin{aligned}
& P(1)=\sum_{i=1}^{E N} P L(i)+\sum_{j=1}^{E N-1} L P(j) \\
& Q(1)=\sum_{i=1}^{E N} Q L(i)+\sum_{j=1}^{E N-1} L Q(j)
\end{aligned}
$$

Step 7: Increment node number $\mathrm{i}=\mathrm{i}+1$

Step 8: If laterals are present at $i^{\text {th }}$ node $\mathrm{NL}(\mathrm{i})>0$, go to step 10 else go to next step

Step 9: Compute $\mathrm{P}(\mathrm{i}+1), \mathrm{Q}(\mathrm{i}+1)$ from equations

$$
\begin{aligned}
& \mathrm{P}(\mathrm{i}+1)=\mathrm{P}(\mathrm{i})-\mathrm{PL}(\mathrm{i})-\mathrm{LP}(\mathrm{i}) \\
& \mathrm{Q}(\mathrm{i}+1)=\mathrm{Q}(\mathrm{i})-\mathrm{QL}(\mathrm{i})-\mathrm{LQ}(\mathrm{i})
\end{aligned}
$$

Then go to step 21

Step 10: Set $\mathrm{k}=0$

Step 11: Increment $\mathrm{k}$ value $\mathrm{k}=\mathrm{k}+1$

Step 12: Compute sum of loss power and load power of $k^{\text {th }}$ lateral at $i^{\text {th }}$ node $B P(i, k), B Q(i, k)$ from equations

$$
B P(\mathrm{i}, \mathrm{k})=\sum_{\mathrm{m}=\mathrm{LB}(\mathrm{i}, \mathrm{k})}^{\mathrm{ENL}(\mathrm{i}, \mathrm{k})} \mathrm{PL}(\mathrm{m})+\sum_{\mathrm{n}=\mathrm{LB}(\mathrm{i}, \mathrm{k})-1}^{\mathrm{ENL}(\mathrm{i}, \mathrm{k})-1} \mathrm{LP}(\mathrm{n}) B \mathrm{Q}(\mathrm{i}, \mathrm{k})=\sum_{\mathrm{m}=\mathrm{LB}(\mathrm{i}, \mathrm{k})}^{\mathrm{ENL}(\mathrm{i}, \mathrm{i})} \mathrm{QL}(\mathrm{m})+\sum_{\mathrm{n}=\mathrm{LB}(\mathrm{i}, \mathrm{k})-1}^{\mathrm{ENL}(\mathrm{i}, \mathrm{k})-1} \mathrm{LQ}(\mathrm{n})
$$

Step13: Compute injected powers at source node of $\mathrm{k}^{\text {th }}$ lateral, $\mathrm{P}(\mathrm{LB}(\mathrm{i}, \mathrm{k}))$ and $\mathrm{Q}(\mathrm{LB}(\mathrm{i}, \mathrm{k}))$ from equations

$\mathrm{P}(\mathrm{LB}(\mathrm{i}, \mathrm{k}))=\mathrm{BP}(\mathrm{i}, \mathrm{k})-\mathrm{LP}(\mathrm{LB}(\mathrm{i}, \mathrm{k})-1)-\mathrm{PL}(\mathrm{LB}(\mathrm{i}, \mathrm{k}))$

$\mathrm{Q}(\mathrm{LB}(\mathrm{i}, \mathrm{k}))=\mathrm{BQ}(\mathrm{i}, \mathrm{k})-\mathrm{LQ}(\mathrm{LB}(\mathrm{i}, \mathrm{k})-1)-\mathrm{QL}(\mathrm{LB}(\mathrm{i}, \mathrm{k}))$

Step14: Calculate injected powers of all nodes of laterals. $\mathrm{P}(\mathrm{x}+1), \mathrm{Q}(\mathrm{x}+1)$ for $\mathrm{x}=\mathrm{LB}(\mathrm{I}, \mathrm{k}) \ldots \mathrm{ENL}-1$ using equations

$\mathrm{P}(\mathrm{x}+1)=\mathrm{P}(\mathrm{x})-\mathrm{PL}(\mathrm{x})-\mathrm{LP}(\mathrm{x})$

$\mathrm{Q}(\mathrm{x}+1)=\mathrm{Q}(\mathrm{x})-\mathrm{QL}(\mathrm{x})-\mathrm{LQ}(\mathrm{x})$

Step15: Compute node voltages of ahead of source node of $\mathrm{k}^{\text {th }}$ lateral using equation

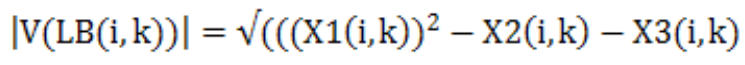

where, $X_{1}(\mathrm{i}, \mathrm{k})=\mathrm{P}(\mathrm{LB}(\mathrm{i}, \mathrm{k})) \mathrm{R}(\mathrm{LB}(\mathrm{i}, \mathrm{k})-1)+\mathrm{Q}(\mathrm{LB}(\mathrm{i}, \mathrm{k})) \mathrm{X}(\mathrm{LB}(\mathrm{i}, \mathrm{k})-1)-0.5|\mathrm{v}(\mathrm{i})|^{2}|\mathrm{v}(\mathrm{i})|^{2}$

$$
\begin{aligned}
& \mathrm{X}_{2}(\mathrm{i}, \mathrm{k})=\left((\mathrm{R}(\mathrm{LB}(\mathrm{i}, \mathrm{k}))-1)^{2}+(\mathrm{X}(\mathrm{LB}(\mathrm{i}, \mathrm{k})-1))^{2}\left((\mathrm{P}(\mathrm{LB}(\mathrm{i}, \mathrm{k})))^{2}+(\mathrm{Q}(\mathrm{LB}(\mathrm{i}, \mathrm{k})))^{2}\right)\right. \\
& \mathrm{X}_{3}(\mathrm{i}, \mathrm{k})=\mathrm{P}(\mathrm{LB}(\mathrm{i}, \mathrm{k})) \mathrm{R}(\mathrm{LB}(\mathrm{i}, \mathrm{k})-1)+\mathrm{Q}(\mathrm{LB}(\mathrm{i}, \mathrm{k})) \mathrm{X}(\mathrm{LB}(\mathrm{i}, \mathrm{k})-1)-0.5|\mathrm{v}(\mathrm{i})|^{2}|\mathrm{v}(\mathrm{i})|^{2}
\end{aligned}
$$

where, $i$ varies from $i=0,1,2, \ldots . ., E N M-1$ 
where $\mathrm{k}=1,2,3,4, \ldots, \mathrm{NL}(\mathrm{i})$ for $\mathrm{k}>0$

Step16: Compute lateral node voltages using equations

$$
|V(x+1)|=\sqrt{ }(A 1) 2-(A 2)(A 3)-A 1
$$

where, $\mathrm{A} 1=\mathrm{P}(\mathrm{x}+1) \mathrm{R}(\mathrm{x})+\mathrm{Q}(\mathrm{x}+1) \mathrm{X}(\mathrm{x})-0.5|\mathrm{~V}(\mathrm{x})|^{2}|\mathrm{~V}(\mathrm{x})|^{2}$

$$
\mathrm{A} 2=(\mathrm{R}(\mathrm{x}))^{2}+(\mathrm{X}(\mathrm{x}))^{2}
$$$$
\mathrm{A} 3=(\mathrm{P}(\mathrm{x}+1))^{2}+(\mathrm{Q}(\mathrm{x}+1))^{2}
$$

where $\quad \mathrm{x}=\mathrm{LB}(\mathrm{i}, \mathrm{p}) \ldots . . \mathrm{ENL}(\mathrm{i}, \mathrm{p})-1$

Step17: If the number of system laterals less than laterals at $\mathrm{i}^{\text {th }}$ node, $\mathrm{k}<\mathrm{NL}(\mathrm{i})$, go to step 10 else go to next step.

Step18: Compute power fed through all nodes, $\mathrm{P}(\mathrm{i}+1)$ and $\mathrm{Q}(\mathrm{i}+1)$ using equations

$$
\begin{aligned}
& \mathrm{P}(\mathrm{i}+1)=\mathrm{P}(\mathrm{i})-\mathrm{PL}(\mathrm{i})-\mathrm{LP}(\mathrm{i})-\sum_{k=1}^{\mathrm{NL}(\mathrm{i})} B P(\mathrm{i}, \mathrm{k}) \\
& \mathrm{Q}(\mathrm{i}+1)=\mathrm{Q}(\mathrm{i})-\mathrm{QL}(\mathrm{i})-\mathrm{LQ}(\mathrm{i})-\sum_{k=1}^{\mathrm{NL}(\mathrm{i})} B Q(\mathrm{i}, \mathrm{k})
\end{aligned}
$$

Step19: Calculate main feeder node voltages $\mathrm{V}(\mathrm{i}+1)$ of a system

$|\mathrm{V}(\mathrm{i}+1)|=\left\{\left[\left(\mathrm{P}(\mathrm{i}+1) \mathrm{R}(\mathrm{i})+\mathrm{Q}(\mathrm{i}+1) \mathrm{X}(\mathrm{i})-0.5|\mathrm{~V}(\mathrm{i}+1)|^{2}\right)^{2}-\left(\left(\mathrm{R}^{2}(\mathrm{i})+\mathrm{X}^{2}(\mathrm{i})\right)\left(\mathrm{P}^{2}(\mathrm{i}+1)+\mathrm{Q}^{2}(\mathrm{i}+1)\right)\right)\right]^{1 / 2}-\left(\mathrm{P}(\mathrm{i}+1) \mathrm{R}(\mathrm{i})+\mathrm{Q}(\mathrm{i}+1) \mathrm{X}(\mathrm{i})-0.5|\mathrm{~V}(\mathrm{i})|^{2}\right)\right\}^{1 / 2}$

Step 20: If voltage at any node is less than 0.92 ( $-8 \%$ of first node) then inject power through Micro Generation into those nodes and go to Step6 else go to step 21

Step 21: If node number $\mathrm{i}<\mathrm{EN}-1$, go to step 7 else go to next step.

Step 22: Compute power losses, LP(i), LQ(i)

Step 23: Compute difference in power

$\mathrm{DP}(\mathrm{i})=\mathrm{LP}(\mathrm{i})-\mathrm{PLoss}(\mathrm{i})$

$D Q(i)=L Q(i)-Q \operatorname{Loss}(i)$ for $i=1,2 \ldots . . E N-1$.

Step 24: If (DP(i)\& DQ(i)) $<\epsilon$ (specified convergence value is 0.000001$)$, go to next step else go to step 3 with iteration increment con $=$ con +1

Step 25: Compute the power loss and voltage profile

Step 26: Calculate voltage regulation at each node with respect to initial voltage

Step 27: Compare voltage profile, voltage regulation and power losses with and without micro- generation.

Step 28: Stop

Based on reduction in power loss compute expected benefit and cost benefit ratio as given in proposed cost benefit algorithm. Pay back period is also calculated from cost benefit ratio for different networks.

\subsection{Algorithm for Cost Benefit Analysis}

Step1: Determine the Active power loss deviation $(\Delta \mathrm{P})$ of the Distribution Network $\Delta \mathrm{P}=\mathrm{P}_{1}-\mathrm{P}_{2}$

where, $\mathrm{P}_{1}=$ Active power loss without Micro Generation

$\mathrm{P}_{2}=$ Active power loss with Micro Generation

Step 2: Calculate the Expected Benefit (EB), by using the formula

$\mathrm{EB}=\Delta \mathrm{P} * 300 * 24 * 16$

where $\quad \Delta \mathrm{P}=$ Active power loss deviation

Step 3: Collect the ratings and cost of inverters, batteries and solar panels

Step 4: Find the investment cost for, $1 \mathrm{~W}$ solar panel cost including inverter and battery $=$ Rs60

Step 5: Now calculate the payback period by using the formula

Payback Period $=\frac{\text { Investment }}{\text { Expected Benefit }}$ 


\subsection{Case study 1: IEEE 34 Bus Network}

This network has 34 buses and 33 branches. From the results obtained, the minimum voltage of the base network and the network with Micro Generation are $\mathbf{0 . 9 2 3 4}$ p.u.and $\mathbf{0 . 9 3 3 9}$ p.u. (at bus 26 )respectively, as shown in table 1. The voltage regulation is improved from 7.66 to 6.61 as shown in table 2. Similarly the active power losses in both the cases are $221.7 \mathrm{KW}, 190.92 \mathrm{KW}$ respectively. The reduction in Active power loss from the result is $13.88 \%$. Fig 1 to 3 .

Table 1. Voltage Profile of IEEE 34 Bus Network

\begin{tabular}{|c|c|c|}
\hline $\begin{array}{l}\text { Bus } \\
\text { No. }\end{array}$ & $\begin{array}{l}\text { Voltage profile of } \\
\text { base network in P.U }\end{array}$ & $\begin{array}{l}\text { Voltage profile in P.U. } \\
\text { with Micro } \\
\text { Generation }\end{array}$ \\
\hline 1 & 1 & 1 \\
\hline 2 & 0.9947 & 0.9951 \\
\hline 3 & 0.9901 & 0.9908 \\
\hline 4 & 0.9838 & 0.9850 \\
\hline 5 & 0.9784 & 0.9801 \\
\hline 6 & 0.9734 & 0.9755 \\
\hline 7 & 0.9704 & 0.9725 \\
\hline 8 & 0.9687 & 0.9708 \\
\hline 9 & 0.9668 & 0.9690 \\
\hline 10 & 0.9660 & 0.9682 \\
\hline 11 & 0.9657 & 0.9679 \\
\hline 12 & 0.9657 & 0.9679 \\
\hline 13 & 0.9898 & 0.9905 \\
\hline 14 & 0.9895 & 0.9902 \\
\hline 15 & 0.9894 & 0.9901 \\
\hline 16 & 0.9894 & 0.9901 \\
\hline 17 & 0.9691 & 0.9718 \\
\hline 18 & 0.9474 & 0.9532 \\
\hline 19 & 0.9436 & 0.9500 \\
\hline 20 & 0.9405 & 0.9475 \\
\hline 21 & 0.9379 & 0.9454 \\
\hline 22 & 0.9350 & 0.9431 \\
\hline 23 & 0.9326 & 0.9412 \\
\hline 24 & 0.9305 & 0.9396 \\
\hline 25 & 0.9256 & 0.9357 \\
\hline 26 & 0.9234 & 0.9339 \\
\hline 27 & 0.9235 & 0.9340 \\
\hline 28 & 0.9702 & 0.9723 \\
\hline 29 & 0.9701 & 0.9722 \\
\hline 30 & 0.9701 & 0.9722 \\
\hline 31 & 0.9658 & 0.9679 \\
\hline 32 & 0.9655 & 0.9677 \\
\hline 33 & 0.9655 & 0.9676 \\
\hline 34 & 0.9655 & 0.9676 \\
\hline
\end{tabular}


Table 2. Results of IEEE 34 Bus Network

\begin{tabular}{|c|c|c|c|c|c|c|c|}
\hline \multicolumn{2}{|c|}{ Power Loss $(\mathrm{kW})$} & \multicolumn{2}{|c|}{$\begin{array}{l}\text { Minimum Voltage in } \\
\text { P.U. }\end{array}$} & \multirow[t]{2}{*}{$\begin{array}{l}\text { Loss Reduction } \\
\text { in } \mathrm{kW}\end{array}$} & \multirow[t]{2}{*}{$\begin{array}{l}\text { \% Loss } \\
\text { Reduction }\end{array}$} & \multicolumn{2}{|c|}{$\begin{array}{l}\text { \%Voltage Regulation } \\
\text { at Min Voltage Bus }\end{array}$} \\
\hline $\begin{array}{l}\text { Base } \\
\text { Network }\end{array}$ & $\begin{array}{l}\text { Proposed } \\
\text { Method }\end{array}$ & $\begin{array}{l}\text { Base } \\
\text { Network }\end{array}$ & $\begin{array}{l}\text { Proposed } \\
\text { Method }\end{array}$ & & & $\begin{array}{l}\text { Base } \\
\text { Network }\end{array}$ & $\begin{array}{l}\text { Proposed } \\
\text { Method }\end{array}$ \\
\hline 221.7 & 190.92 & 0.9234 & 0.9339 & 30.78 & 13.88 & 7.66 & 6.61 \\
\hline
\end{tabular}

Table 3. Voltage Profile of EPDCL 34 Bus Network

\begin{tabular}{|c|c|c|}
\hline BUS No & $\begin{array}{l}\text { Voltage profile of base } \\
\text { network in P.U. }\end{array}$ & $\begin{array}{l}\text { Voltage profile in } \\
\text { P.U. with Micro } \\
\text { Generation }\end{array}$ \\
\hline 1 & 1 & 1 \\
\hline 2 & 0.987 & 0.9886 \\
\hline 3 & 0.9808 & 0.9833 \\
\hline 4 & 0.9695 & 0.9736 \\
\hline 5 & 0.9639 & 0.9688 \\
\hline 6 & 0.9584 & 0.964 \\
\hline 7 & 0.9507 & 0.958 \\
\hline 8 & 0.9394 & 0.9491 \\
\hline 9 & 0.9379 & 0.9479 \\
\hline 10 & 0.9349 & 0.9455 \\
\hline 11 & 0.9348 & 0.9455 \\
\hline 12 & 0.9348 & 0.9455 \\
\hline 13 & 0.9807 & 0.9832 \\
\hline 14 & 0.9805 & 0.9829 \\
\hline 15 & 0.9803 & 0.9827 \\
\hline 16 & 0.9802 & 0.9827 \\
\hline 17 & 0.9802 & 0.9827 \\
\hline 18 & 0.9533 & 0.959 \\
\hline 19 & 0.9505 & 0.9563 \\
\hline 20 & 0.9475 & 0.9532 \\
\hline 21 & 0.9465 & 0.9522 \\
\hline 22 & 0.9458 & 0.9516 \\
\hline 23 & 0.9458 & 0.9516 \\
\hline 24 & 0.9351 & 0.9457 \\
\hline 25 & 0.9313 & 0.9427 \\
\hline 26 & 0.929 & 0.9409 \\
\hline 27 & 0.9269 & 0.9392 \\
\hline 28 & 0.9258 & 0.9383 \\
\hline 29 & 0.9258 & 0.9383 \\
\hline 30 & 0.9296 & 0.9414 \\
\hline 31 & 0.9265 & 0.9388 \\
\hline 32 & 0.9238 & 0.9368 \\
\hline 33 & 0.9228 & 0.9359 \\
\hline 34 & 0.9228 & 0.9359 \\
\hline
\end{tabular}


Table 4. Results of EPDCL 34 Bus Network

\begin{tabular}{|c|c|c|c|c|c|c|c|}
\hline \multicolumn{2}{|c|}{ Power Loss(kW) } & \multicolumn{2}{|c|}{$\begin{array}{c}\text { Minimum Voltage in } \\
\text { P.U. }\end{array}$} & \multirow{2}{*}{$\begin{array}{l}\text { Loss Re- } \\
\text { duction } \\
\text { in } \mathrm{kW}\end{array}$} & \multirow{2}{*}{$\begin{array}{l}\text { \% Loss } \\
\text { Reduction }\end{array}$} & \multicolumn{2}{|c|}{$\begin{array}{l}\% \text { Voltage Regulation } \\
\text { at Min Voltage Bus }\end{array}$} \\
\hline $\begin{array}{c}\text { Base } \\
\text { Network }\end{array}$ & $\begin{array}{c}\text { Proposed } \\
\text { Method }\end{array}$ & $\begin{array}{c}\text { Base } \\
\text { Network }\end{array}$ & $\begin{array}{c}\text { Proposed } \\
\text { Method }\end{array}$ & & & $\begin{array}{c}\text { Base } \\
\text { Network }\end{array}$ & $\begin{array}{l}\text { Proposed } \\
\text { Method }\end{array}$ \\
\hline 93.7529 & 67.6125 & 0.9228 & 0.9359 & 26.1404 & 27.88 & 7.72 & 6.41 \\
\hline
\end{tabular}

Table 5. Cost Benefit Ratio

\begin{tabular}{|c|c|c|c|c|c|c|c|c|}
\hline \multirow{2}{*}{$\begin{array}{l}\text { Type of } \\
\text { Network }\end{array}$} & \multicolumn{2}{|c|}{ Power Loss in $\mathrm{kW}$} & \multirow{2}{*}{$\begin{array}{l}\text { Reduction } \\
\text { of Loss in } \\
\mathrm{kW}\end{array}$} & \multirow{2}{*}{$\begin{array}{l}\text { \%Reduction } \\
\text { in loss }\end{array}$} & \multirow{2}{*}{$\begin{array}{c}\text { Expected } \\
\text { Benefit }\end{array}$} & \multirow{2}{*}{$\begin{array}{l}\text { Investment } \\
\text { (INR) }\end{array}$} & \multirow{2}{*}{$\begin{array}{l}\text { Cost to } \\
\text { Bene- } \\
\text { fit Ratio }\end{array}$} & \multirow{2}{*}{$\begin{array}{c}\text { Pay Back Period } \\
\text { (years) }\end{array}$} \\
\hline & $\begin{array}{c}\text { Base } \\
\text { Network }\end{array}$ & $\begin{array}{c}\text { Proposed } \\
\text { Method }\end{array}$ & & & & & & \\
\hline 32 (EPDCL) & 91.4332 & 67.0126 & 24.421 & 26.71 & $28,13,184$ & $4,55,94,000$ & 16.2 & 16.2 \\
\hline 34(EPDCL) & 93.7529 & 67.6125 & 26.1404 & 27.88 & 3011374 & 65495220 & 21.74 & 21.74 \\
\hline 33(IEEE) & 202.67 & 129.1729 & 73.497 & 36.26 & $84,66,885$ & $7,66,68,300$ & 9.05 & 9.05 \\
\hline
\end{tabular}

A 34 bus network is considered as shown in fig 4.This network has 34 buses and 33 branches, from the results obtained, the minimum voltage of the base network and the network adopted with Micro Generation are $\mathbf{0 . 9 2 2 8}$ p.u. and $\mathbf{0 . 9 3 5 9}$ p.u. (at bus 34 ) respectively, as shown in table 3. The \%voltage regulation is improved from 7.72 to 6.58 as shown in table 4. Line and Load data is presented in Appendix 1. Similarly the active power losses in both the cases are $93.7529 \mathrm{~kW}, 67.6125 \mathrm{~kW}$ respectively. The reduction in Active power loss from the result is obtained as $27.88 \%$.

Fig.1. Voltage Profile Curve of IEEE 34 Bus Network

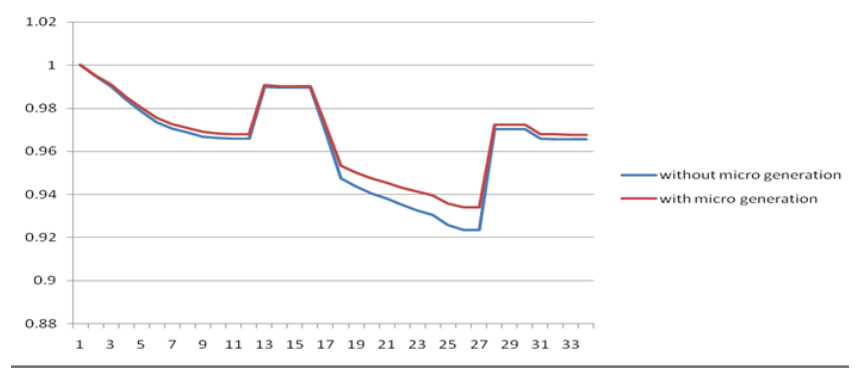

Fig.3. Active Power Loss Curve of IEEE 34 bus system

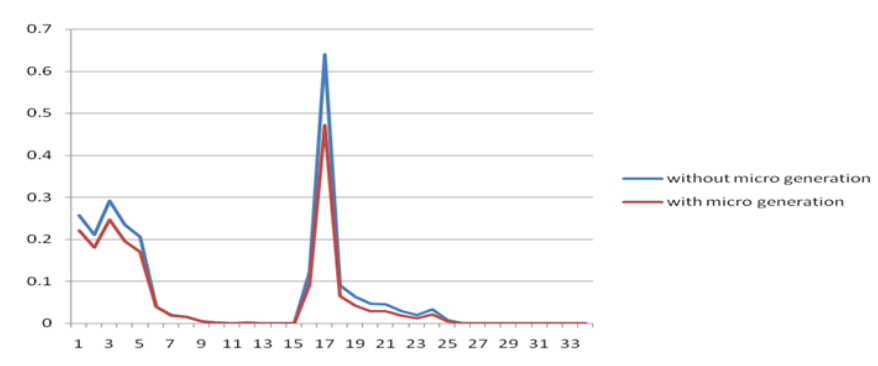

Fig.2. Voltage Regulation Curve of IEEE 34 Bus Network

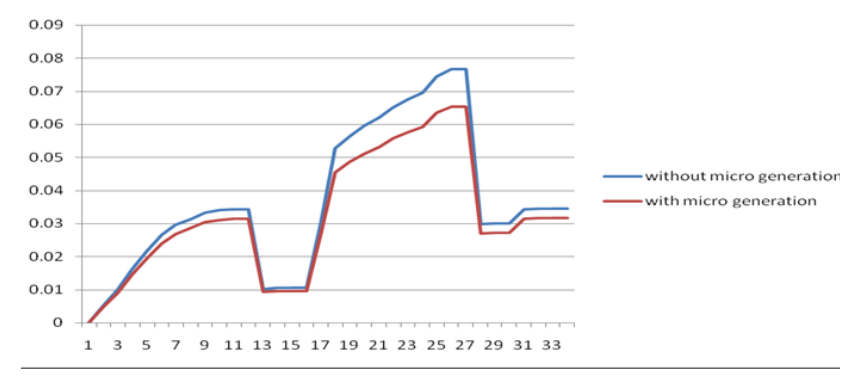

Fig.4. EPDCL 34 Bus Network

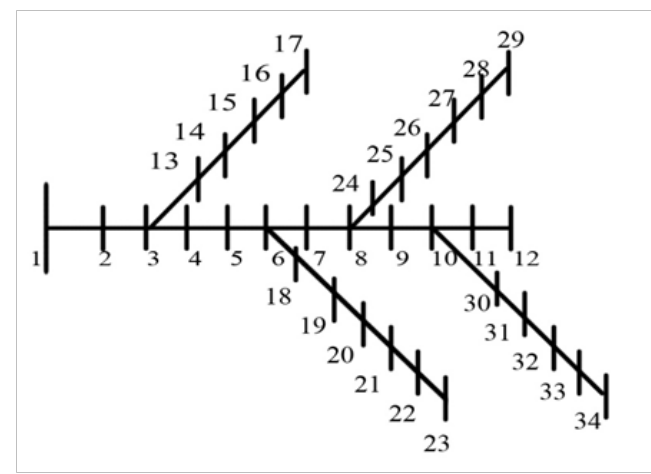


Fig.5. Voltage Profile Curve of EPDCL 34 Bus Network

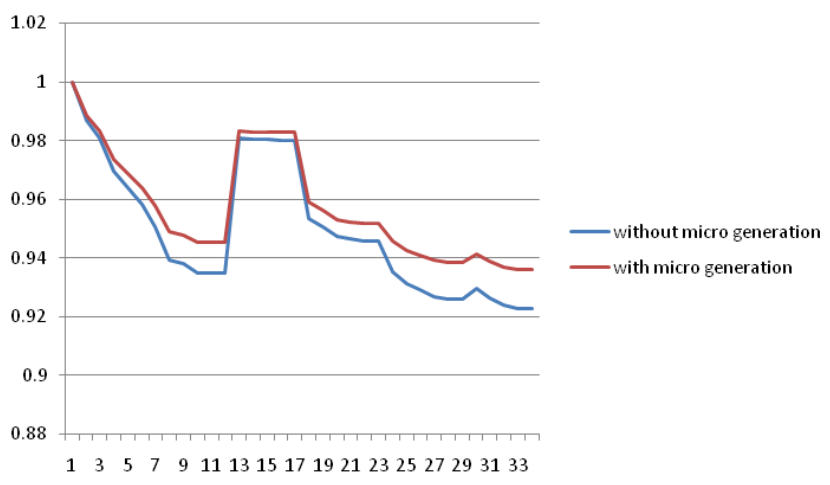

Fig.7. Active Power Loss Curve of EPDCL 34 bus system

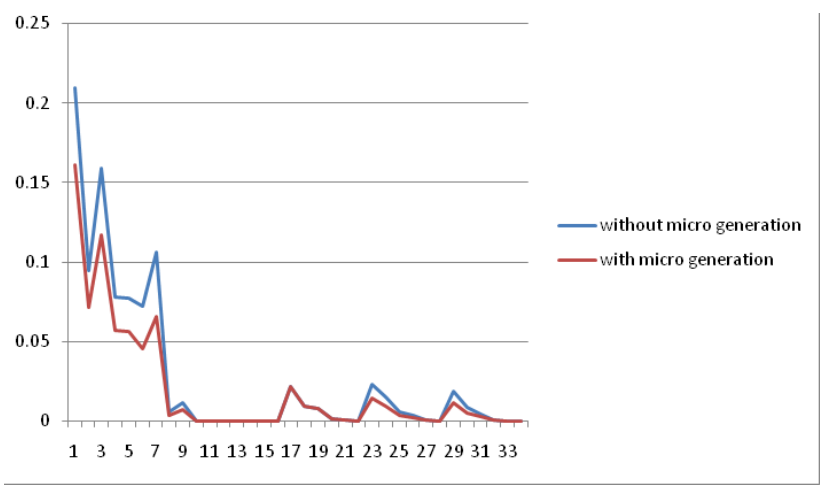

Fig.6. Voltage Regulation Curve of EPDCL 34 Bus Network

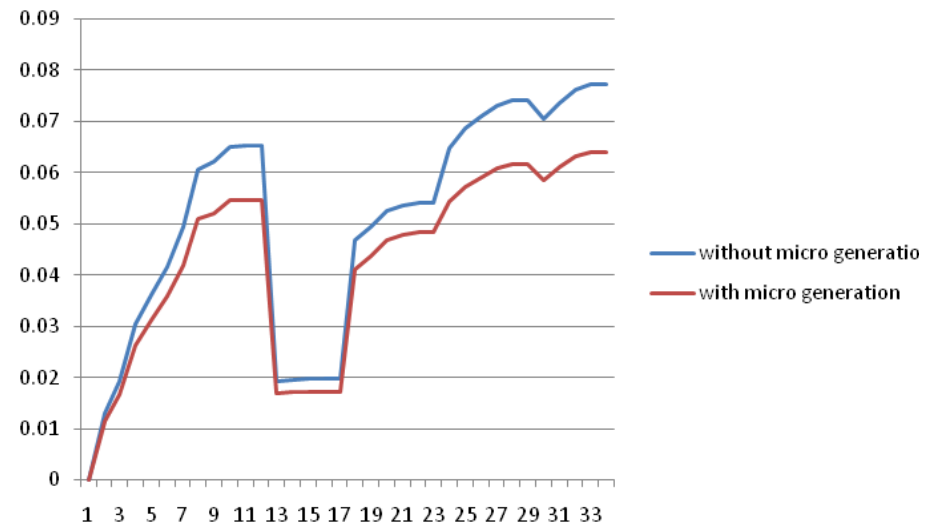

\section{Conclusion}

An attempt has been made to study the impact of using Micro Generation of solar power to augment the regular power supply is made, which is reliable and eco-friendly. It is observed that the power loss is reduced and voltage profile is improved in the radial distribution network in both cases. The average voltage of radial distribution network with Micro Generation is increased by $1.584 \%$ and the total power loss with Micro Generation is decreased by $27.52 \%$ for the existing network compared to the base network. From the results of payback period it is observed that for IEEE 33 bus network the payback period is comparatively low. From Table 5 it is inferred that the payback period is 9.05 years. Though the payback periods are not very encouraging now, two factors make this alternative feasible: The cost of solar panels and supporting equipment are reducing considerably as the demand is increasing and the cost of generation of energy is negligible. The cost of fossil fuels is increasing as they are depleting which offsets the cost of solar panels. Considering these facts the solar Micro Generation has promising future in countries like India where solar energy is abundant.

\section{References}

1 All India region wise generating installed capacity of power (2011) Central Electricity Authority, Ministry of power, Government of India.

2- Chen TH, Chen M, Hwang KJ, Kotas P and Chebli EA (1981) Distribution system power flow analysis - A rigid approach. IEEE Trans. on Power Delivery. 6 (3),1146-1152.

3. Distribution automation: a practical tool for shaping a more profitable Future Special report (1986) Electrical World. pp: 43-50, Dec.

4- Power sector at a glance: All India data (2011) Ministry of power, Government of India. 
Sethi, Nitin (2009) India targets 1,000Mw solar Power in 2013. Times of India. Nov.18.

6• Vempati N, Shoults RR, Chen MS and Schwobel L (1987) Simplified feeder modeling for load flow calculations. IEEE Trans. Power Systems. 2 (1), 168-174.

\section{Appendix 1. Line and Load data of EPDCL 34 Bus}

\begin{tabular}{|c|c|c|c|c|}
\hline Bus No & $R(\Omega)$ & $X(\Omega)$ & $\mathbf{P}(\mathbf{k W})$ & Q(kVAR) \\
\hline 1 & 0.908 & 0.314 & 0 & 0 \\
\hline 2 & 0.454 & 0.157 & 72.25 & 44.8 \\
\hline 3 & 0.908 & 0.314 & 72.25 & 44.8 \\
\hline 4 & 0.454 & 0.157 & 10.8375 & 6.72 \\
\hline 5 & 0.454 & 0.157 & 10.8375 & 6.72 \\
\hline 6 & 0.908 & 0.314 & 10.8375 & 6.72 \\
\hline 7 & 1.362 & 0.471 & 10.8375 & 6.72 \\
\hline 8 & 0.454 & 0.157 & 10.8375 & 6.72 \\
\hline 9 & 0.908 & 0.314 & 10.8375 & 6.72 \\
\hline 10 & 0.454 & 0.157 & 10.8375 & 6.72 \\
\hline 11 & 0.2724 & 0.0942 & 10.8375 & 6.72 \\
\hline 12 & 0.2724 & 0.0942 & 10.8375 & 6.72 \\
\hline 13 & 0.908 & 0.314 & 10.8375 & 6.72 \\
\hline 14 & 0.908 & 0.314 & 10.8375 & 6.72 \\
\hline 15 & 0.454 & 0.157 & 10.8375 & 6.72 \\
\hline 16 & 0.681 & 0.2355 & 10.8375 & 6.72 \\
\hline 17 & 1.362 & 0.471 & 10.8375 & 6.72 \\
\hline 18 & 0.908 & 0.314 & 72.25 & 44.8 \\
\hline 19 & 1.362 & 0.471 & 72.25 & 44.8 \\
\hline 20 & 0.681 & 0.2355 & 72.25 & 44.8 \\
\hline 21 & 0.908 & 0.314 & 72.25 & 44.8 \\
\hline 22 & 1.0896 & 0.3768 & 72.25 & 44.8 \\
\hline 23 & 0.908 & 0.314 & 72.25 & 44.8 \\
\hline 24 & 1.0896 & 0.3768 & 115.6 & 71.67 \\
\hline 25 & 0.9988 & 0.3454 & 115.6 & 71.67 \\
\hline 26 & 1.362 & 0.471 & 72.25 & 44.8 \\
\hline 27 & 1.589 & 0.5495 & 72.25 & 44.8 \\
\hline 28 & 1.4074 & 0.4867 & 72.25 & 44.8 \\
\hline 29 & 1.6798 & 0.5809 & 72.25 & 44.8 \\
\hline 30 & 1.362 & 0.471 & 72.25 & 44.8 \\
\hline 31 & 1.6798 & 0.5809 & 72.25 & 44.8 \\
\hline 32 & 1.4074 & 0.4867 & 72.25 & 44.8 \\
\hline 33 & 1.589 & 0.5495 & 72.25 & 44.8 \\
\hline 34 & 0 & 0 & 72.25 & 44.8 \\
\hline
\end{tabular}

